\title{
An Efficient Link Aware Route Selection Algorithm for WiMAX Mobile Multi-hop Relay Networks
}

\author{
N. Satiman \\ UTM-MIMOS CoE \\ Faculty of Electrical \\ Engineering \\ Universiti Teknologi Malaysia
}

\author{
A.I.A. Zamani \\ UTM-MIMOS CoE \\ Faculty of Electrical \\ Engineering \\ Universiti Teknologi Malaysia
}

\author{
N. Fisal \\ UTM-MIMOS CoE \\ Faculty of Electrical \\ Engineering \\ Universiti Teknologi Malaysia
}

\author{
S.K.S. Yusof \\ UTM-MIMOS CoE \\ Faculty of Electrical \\ Engineering \\ Universiti Teknologi Malaysia
}

\author{
N.N.M.I. Maa'rof \\ UTM-MIMOS CoE \\ Faculty of Electrical \\ Engineering \\ Universiti Teknologi Malaysia
}

\begin{abstract}
Multi-hop relay technology is designed to provide capacity enhancement and coverage extension for wireless broadband access system such as WiMAX and LTE-Advanced. However, overall system performances worsen as the number of hop increases. For this reason, resource control function specifically route selection problem should be tackled precisely so that better system performance can be achieved. Route selection or routing is a process to identify the best route to deliver information from source to destination by considering the constraints of available radio resource of the route. In this paper, we proposed a new route selection scheme named as Link Aware Route Selection Scheme (LARSS) for WiMAX Mobile Multi-hop Relay Networks aiming at maximizing network throughput and minimizing end-to-end delay. The proposed scheme exploits link quality and hop count as route metric. We conducted simulation study to evaluate the performance of the proposed scheme. Through the simulation, our proposed scheme outperformed the existing scheme in term of throughput and end-to-end delay.
\end{abstract}

\section{General Terms \\ Algorithms}

\section{Keywords}

Route selection, multi-hop relay, WiMAX, link quality, cross layer

\section{INTRODUCTION}

Wider bandwidth at higher operating frequency is expected to be used in order to fulfill the demand upon high-speed multimedia services with Quality of Service (QoS) guarantee. Unfortunately, the use of higher frequency might results in significant loss of signal strength along the propagation path. In brief, it will cause coverage limitation and offer low data rate at to the cell edge users. Multi-hop relaying technology is seen as an efficient solution that could enhances coverage so as improves system throughput without a need to deploy expensive base station. Motivated from this scenario, several wireless system has deployed multi-hop communication on their system such as cellular network, mesh network, sensor network etc.

In WiMAX system, the new task group called IEEE $802.16 \mathrm{j}$ Mobile Multihop Relay (MMR) [1] is formed to construct the extension of IEEE 802.16e that includes multi-hop relaying capabilities to extend the coverage area and improve throughput of mobile WiMAX network while ensuring compatibility with point-to-multipoint (PMP) mode. In $802.16 \mathrm{j}$, relay station (RS) is introduced as an intermediate node to relay the data between base station (BS) and subscriber station (SS)/mobile station (MS). The introduction of RS provides several advantages to the wireless system performance. First, RS can extend the coverage area. Thus, it would allow those users that located at remote area to be served. In addition, RS can help to reach users which placed at shadows areas where direct communication with the BS is infeasible. Second, RS can increase the network throughput by improving the signal-to-noise ratio (SNR). Typically, relay link is shorter than direct link between BS and MS, thus, result in less signal attenuation in transmission path. Consequently, SNR along the path is improved.

Recently, multi-hop relay network brings much research interest such as resource allocation, scheduling and routing strategy. It is expected that system performance in multi-hop relay environment worsen as number of hops increases. In fact, many work on this research area limits to two hop communication since this is the only scenario that can guarantee performance enhancement. However, the purpose of having relay for serving users in remote area cannot be achieved if data transmission is limited to two hops. For this reason, it is worth to design the resource control function for multi-hop relay network that support more than two hops. The most significant challenge in 
multi-hop relay network is to achieve system performance at least comparable to two hops communication while guaranteeing user's Quality of Service (QoS). In this paper, we are trying to tackle route selection problem for WiMAX MMR networks. Route selection or routing is a process to identify the best route to deliver information from source to destination by considering the constraints of available radio resource of the route. Our aim is to provide the efficient route for data transmission which offers maximum throughput and minimum latency. The contribution of this paper involves the developing of route selection algorithm with novel path cost. In addition, we considered extended number of hops while much related works usually limits to two hops only. The remainder of the paper is organized as follows. Section 2 provides several research works that related to this work. In section 3, we present an overview of WiMAX MMR network. Network model and the proposed route selection algorithm are presented in section 4 and 5, respectively. In section 6 , we present simulation results of proposed work and finally, section 7 concludes the paper.

\section{RELATED WORKS}

From literature, several routing schemes have been proposed for throughput improvement in multi-hop relay network. K. Wang et.al [2] proposed route selection based on optimum throughput. They only focus on two-hop networks. Thus, users only need to select communication with MRBS directly or through RS. Routing scheme that take into account maximization of radio resource utilization is proposed by [3]. They manage to show their proposed scheme is better than link-quality based scheme. However, their scheme is limit to two hops only. D. J. Son et.al [4] proposes path selection scheme based on spectral efficiency and traffic load for IEEE 80216j network. In their scheme, they were aiming at higher throughput as well as higher fairness among MSs. Simulation study shows that their scheme had improve throughput performance compared to others work. However, their throughput performance might degrade as there is a possibility that the selected path consume large number of hops. In [5], K.P. Shih et.al introduces new metric called SEL to evaluate the possible optimal path. SEL mainly considers link spectral efficiency and link load. However, this work focusing on two hop relay network only.

In [6], the author had proposed new approach for RS selection in IEEE802.16j network. The route selection is based on expected link throughput. In this scheme, path with high link throughput is selected as optimal path. Simulation results had shown that this scheme achieved high throughput when the number of hop increase to two. When the hop is three, the throughput showed a decrement but is still acceptable. However, as the number of hops increase to four and above, the throughput drops significantly. It might be due to the centralized approach used in this scheme. Work done by [7] use channel condition as their metric for route selections in WiMAX MMR networks. In this work, they manage to show that channel condition give significant impact on throughput performance. However, by simply depend on channel condition cannot provides best system performance. For instance, in the MMR network, the chosen path has better channel condition. Unfortunately, that chosen path has larger number of hops, thus the latency performance could be worsen since by adding the hops, it will introduce an additional delay.

\section{OVERVIEW OF WIMAX MOBILE MULTI-HOP RELAY NETWORKS (MMR)}

A typical model of WiMAX-based multi-hop relay network is shown in Figure 1. The network consists of one Multi-hop Relay Base Station (MRBS), several RS and several MS/SS. In MMR network, data is transmitted from the source to the destination through intermediate RS. The concept of relaying clearly shows that communication between the users must go through the MRBS first. Thus, users cannot directly communicate with each other. Generally, relay cannot transmit and receive at the same time using the same channel. In other words, relay works in half-duplex mode. Two kinds of links defined in relay network include access link and relay link. Access link describes the physical channel between source node and the relay node while relay link constitutes physical channel between relay node and destination node.

Relaying method can be classified into three categories, depending on how the received signal is processed. Those categories include amplify-and-forward (AF), decode-andforward (DF) and estimate-and-forward (EF). In AF, relay node acts as a repeater and it will simply amplifying the received signal and forwarding the signal to the destination. The RS does not have to know the content of the receiving packet, thus the processing time could be shortened. However, if this relaying method is applied to a multi-hop relay network with $n>2$, where $n$ is the number of hop, the signal and noise will be amplified resulting in performance degradation at the destination. As for DF, relay node demodulates and decodes the received signal before forwarding it to the destination. DF RS guarantees messages can be received at destination and the processing time at the RS with this relaying method is longer. In $\mathrm{EF}$, the relay node does not decode the input data. It sends an estimate of its channel output to the destination. This estimation will be used by the destination while coding the actual direct link signal [8].

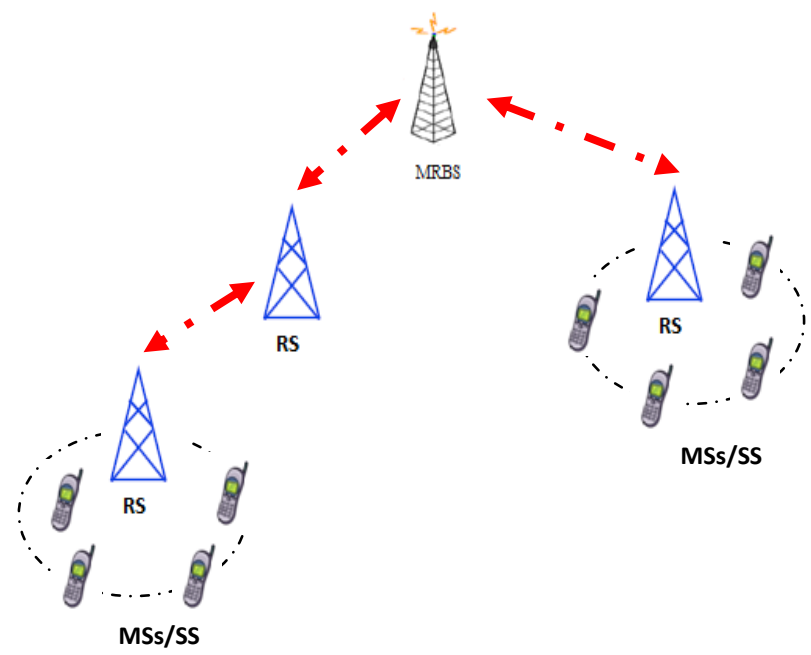

Fig 1: An exemplary scenario of WiMAX MMR networks 
The IEEE802.16j draft standard defines two modes of RS which is transparent and non-transparent. In transparent mode [7], RSs do not forward framing information such as UCD/DCD or DLMAP/UL-MAP; hence, do not increase the coverage area of the wireless access system. For this reason, the main use case for this RS mode is to assist in increasing capacity within the MRBS coverage area which means the MS should be placed in the transmission range of MRBS. This type of RS only operates in a centralized scheduling mode and for topology limits to two hops. In non-transparent mode [9], the RSs create their own framing information or forward those provided by the MRBS depending on the scheduling approach (i.e., distributed or centralized). The purpose of this type of RSs is mainly to provide extended coverage. In contrast, the transmission of the framing information can result in high interference between neighboring RSs. Thus, the capacity enhancement that can be achieved using these relays is limited. Non-transparent relays can operate in either centralized or distributed scheduling mode with topologies larger than two hops.

\section{NETWORK MODEL}

In this work, we focus on the performance of uplink transmission for WiMAX MMR networks. We consider nontransparent RS mode which reflecting that the network model used in this work supports more than two hops communication. Without loss of generality, the system is assumed using timeslotted resource allocation method and all links in the system operate on the same frequency band. In order to meet the IEEE 802.16 specification, data transmission among stations is synchronized on frame basis. A frame comprises of several OFDMA symbols and subchannel. One OFDMA symbol and one subchannel jointly serve as a basic time unit for transmission in the system. We assume the system uses time division transmit and receive relay mode supporting multi-frame structure [10]. Therefore, once each station receives data from its subordinate, i.e. child node, it must wait for one frame time to forward those data to its superordinate stations, i.e. the parent node. Fig. 2 illustrates the DL and UL communication between each station for three hop relay networks supporting multi-frame structure. As seen in Fig.2, MRBS send packet to $1^{\text {st }}$-tier RS in DL Relay Zone of frame $(\mathrm{N})$. Then, $1^{\text {st }}$-tier RS will wait for frame $(\mathrm{N}+1)$ to forward the received packet to $2^{\text {nd }}-$ tier RS. For $\mathrm{UL}$, it follows the same procedure as DL.

In our system, a communication link between two nodes is established if and only if the receiving node is within the maximum transmission range of transmitting node. To estimate the received signal power at the receiving node, we exploit two rays ground model which is expressed by follows [11],

$$
P_{r}=\left(P_{t} * G_{t} * G_{r} * h_{t} * h_{r}\right) / d^{4}
$$

where $P_{t}$ is transmit power, $G_{t}$ is transmitter antenna gain, $G_{r}$ is receiver antenna gain, $h_{t}$ is transmitter antenna height, $h_{r}$ is receiver antenna height and $d$ is Euclidean distance between transmitter and receiver. From (1), then we can compute SNR as follows [12],

$$
\begin{aligned}
& S N R=P_{r}-10 \log \left(\left[F_{s} * N_{\text {used }}\right] / N_{F F T}\right)+114+10 \log R- \\
& \text { ImpLoss }-N F
\end{aligned}
$$

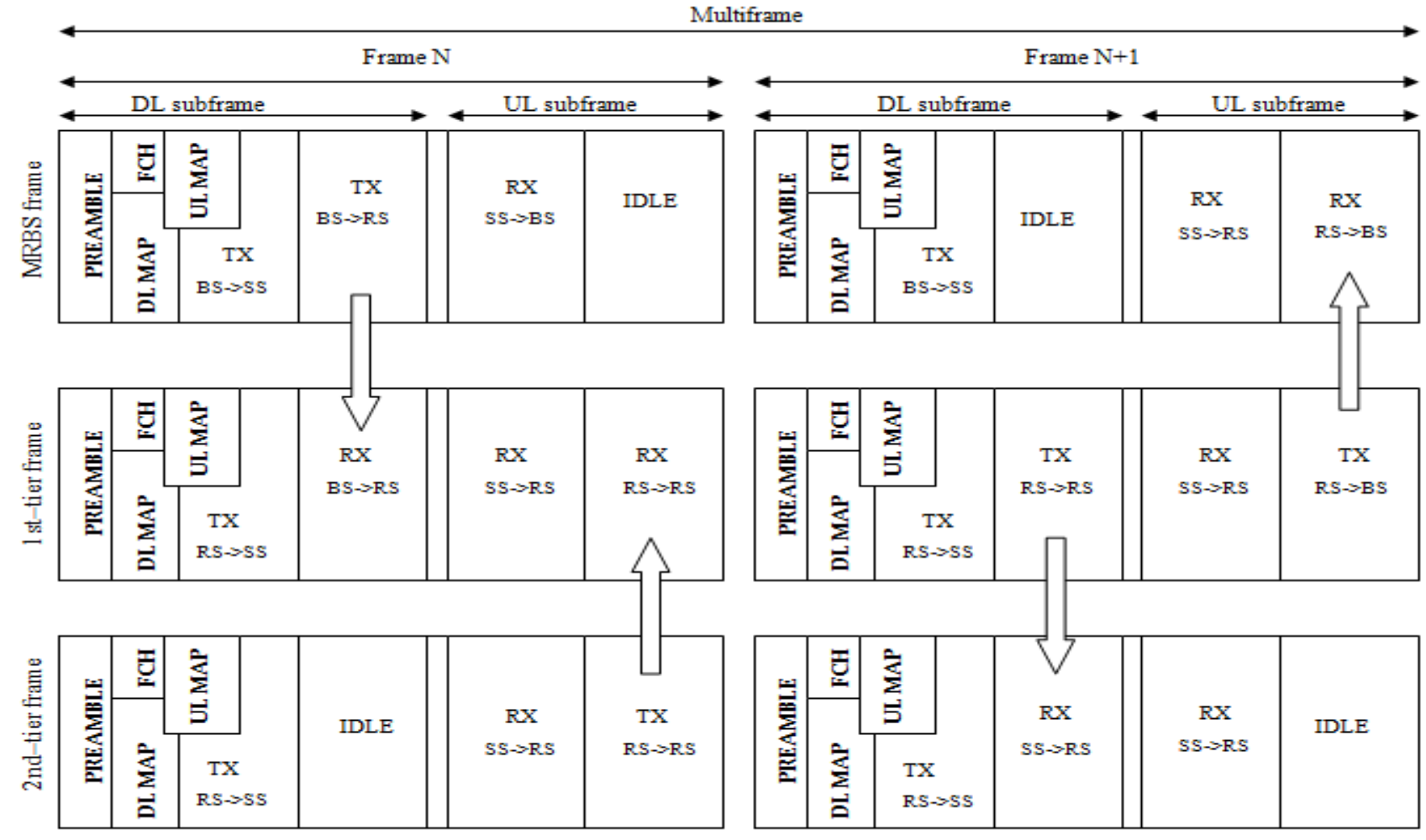

Fig. 2. OFDMA frame structure for three hop relay networks with multi-frame structure 
where $P_{r}$ is received power, $F_{S}$ is sampling frequency in Mhz, $N_{\text {used }}$ is number of used subcarrier, $N_{F F T}$ is number of Fast Fourier Transform subcarrier, $R$ is repetition factor, ImpLoss is implementation loss and $N F$ is noise figure. Note that to ensure the information delivered through a link is decoded correctly; the received SNR should be above the receiver sensitivity. For this reason, any link which is below receiver sensitivity, i.e. $5 \mathrm{~dB}$ is considered as disconnected. SNR quite important in WiMAX system as this value reflect the link quality so as defines modulation schemes and coding rates to be used in that link. The higher the SNR, the less robust modulation is used. Table 1 show the relationship of SNR, modulation scheme and coding rates. According to the table, if received SNR is $12 \mathrm{~dB}$, so the modulation scheme that should be used is 16QAM with coding rate $1 / 2$. Once the modulation scheme and coding rate is chosen, the link spectral efficiency in term of bytes per slot can be obtained. Link spectral efficiency is defined as the amount of data that can be transmitted over a given bandwidth on a link. Equation (3) expressed on how to compute the link spectral efficiency [13].

$$
S E=N_{\text {symbol }} * N_{\text {sub }} * C R * \log m
$$

where $N_{\text {symbol }}$ is number of OFDMA symbol, $N_{\text {sub }}$ is number of subcarrier, $C R$ is coding rate and $m$ is modulated symbol. The link spectral efficiency for each modulation scheme and coding rates(MCS) is also illustrates in Table 1. From the table, we can observe that as SNR increase, the link spectral efficiency increase which means more bits can be transmitted per unit time.

Table 1. The relationship of attainable spectral efficiency of one slot in OFDMA frame for different type of modulation and coding scheme

\begin{tabular}{ccccc}
\hline $\begin{array}{c}\text { Modulation } \\
\text { type }\end{array}$ & $\begin{array}{c}\text { Coding } \\
\text { rate }\end{array}$ & SNR(dB) & Bits/symbol & Bytes/slot \\
\hline QPSK & $1 / 2$ & 5.0 & 2 & 6 \\
& $3 / 4$ & 8.0 & 2 & 9 \\
16QAM & $1 / 2$ & 10.5 & 4 & 12 \\
& $3 / 4$ & 14.0 & 4 & 18 \\
64QAM & $1 / 2$ & 16.0 & 6 & 18 \\
& $2 / 3$ & 18.0 & 6 & 24 \\
& $3 / 4$ & 20.0 & 6 & 27 \\
\hline
\end{tabular}

\section{PROPOSED ROUTE SELECTION SCHEME}

\subsection{Route Metric}

In this paper, we proposed a new route selection metric based on link quality and hop count. The reason of using link quality is that this parameter reflects the link spectral efficiency that is achieved in a link. The higher the SNR, the better link spectral efficiency is provided. In addition, link quality signifies the physical distance between nodes. Better link quality illustrates that the distance between two nodes is small. Thus, the propagation delay can be reduced so as helps in minimizing the latency or end-to-end (ETE) delay.

When working on the network with more number of hops, ETE delay performance could be worsening. For this reason, hop count will let the system to work on feasible number of hops. However, depending only on the hop count cannot guarantee the best system performance. In fact, shortest hop count might include poor link channel on it. Thus, degrades the system performance. For this reason, we integrate hop count and link quality with the aim to obtain an optimal route.

\subsection{Link Aware Route Selection Metric}

The proposed LARSS performed route selection based on link quality and hop count. Initially, we identify all possible paths leading to MRBS. In finding available path, we introduce new signaling message called CNTRL_MSG. CNTRL_MSG contains hop count, SNR value and route cost for previous link. This message first initiate by MRBS and one hop neighbor of MRBS, i.e. RS, will received this message. Upon receiving CNTRL_MSG, RS first check either it has received this message previously or not. If yes, RS will drop this message immediately. If not, RS proceed with updating the route cost. The route cost is defined as follows,

$$
\operatorname{cost}_{i}(r)=\operatorname{cost}_{i-1}(r)+\left[\alpha * h_{i}+\beta * S N R_{i}\right]
$$

Where $h_{i}$ is hop count at node $i, S N R_{i}$ is the minimum SNR value at node $i, \alpha$ and $\beta$ are the weight for each parameter. In our work, we set $\alpha$ and $\beta$ as 1 . Note that the minimum SNR value is obtained by comparing the SNR value of the previous link and SNR value of the current link. After computing route cost, current RS then forwards this message to their 1-hop neighbors. This process is repeating until the receive RS is an access RS, i.e. RS that attach with MSs. While an access RS receive the CNTRL_MSG, it will select the optimal path by means of checking the route cost of all available path. For simplicity, we define available path as $\mathrm{P}=\{P(1), P(2), \ldots, P(r)\}$ where $r$ is number of available path. Each path is associated with a route cost which can be obtained by using equation (4). For instance, $\operatorname{cost}(1)$ is a route cost for $P(1)$. Thus, optimal path can be determined by

$$
O P=\min \{\operatorname{cost}(1), \operatorname{cost}(2), \ldots, \operatorname{cost}(r)\}
$$

From (5), it can be observe that by selecting the minimum cost among all candidate, it reflect a path with smaller number of hops and better channel quality. Thus, lead to a better system performance. To achieve high throughput and less ETE delay, the route should provide smaller hops and exploit less robust modulation and coding scheme. Our proposed LARSS do looking at those features while making a selection. On the other hand, LARSS offers maximum achievable throughput by eliminating bottleneck links.

\section{SIMULATION RESULTS}

To evaluate the performance of proposed scheme, we have conducted simulation study using NCTUns-6.0 network simulator. The objective of our simulation work is to investigate the performance of the proposed scheme in term of throughput and ETE delay in non-transparent mode of WiMAX MMR network. The default simulation scenario consists of 1 MRBS, $10 \mathrm{RSs}$ and 5 MSs. RSs is randomly located in the network. Unsolicited Grant Services (UGS) traffic is generated by MSs and maximum sustained rate for each MS is set to $64 \mathrm{Kbps}$. Two 
ray ground is adopted in the simulation. Table 2 summaries the simulation parameter used in this work.

We introduce shortest path scheme in order to compare with the proposed scheme. The shortest path scheme makes a path selection highly depend on hop count. In this scheme, path with lowest hop count is preferable. We specified two performance metrics to assess the performance of the proposed scheme. Throughput is defined as number of bits received per unit time while average ETE delay is defined as the time between packets is generated to the time packets is received at destination (i.e. MRBS).

Table 2. List of simulation parameters

\begin{tabular}{cc}
\hline Parameter & Value \\
\hline Channel Bandwidth & $10 \mathrm{Mhz}$ \\
Frequency & $2.3 \mathrm{Ghz}$ \\
$\mathrm{N}_{\text {used }}$ & 720 \\
FFT size & 1024 \\
Cyclic Prefix & $1 / 8$ \\
BS transmit power & $43 \mathrm{dBm}$ \\
RS transmit power & $43 \mathrm{dBm}$ \\
MS transmit power & $35 \mathrm{dBm}$ \\
Frame Duration & $5 \mathrm{~ms}$ \\
Symbol duration & $102.9 \mathrm{us}$ \\
Payload size & $128 \mathrm{byte}$ \\
\hline
\end{tabular}

\subsection{Throughput}

Fig. 3 shows the throughput performance of proposed scheme compared to shortest path scheme. As seen in Figure 3, the throughput increases as traffic load increases. However, when the traffic load had reached $40 \mathrm{Kbps}$ and above, the throughput performance turns out to be saturated. The reason behind this outcome is that the traffic load is almost reached the maximum bit rate of each user. Thus, to ensure the transmission is not over the maximum bit rate, the data rate is limited to certain value. Hence, the throughput is saturated to $250 \mathrm{Kbps}$ although the load is increased. The proposed scheme outperformed the shortest path scheme by about $6.7 \%$. This outcome had proven that the path with shortest hop count does not promises better throughput performance. The proposed scheme offers better performance since the choice of path considers link quality and node distance. While considering these two parameters, the selected path will consists of links with better SNR which then provides higher data rate. As a result, throughput performance is better regardless of number of hops involved in the path.

\subsection{End to end Delay}

Average ETE delay performance comparison is shown in Figure 4. From this figure, it can be seen that light traffic does not give any effect to latency performance since it remains around $50 \mathrm{~ms}$ as traffic load increases. However, heavy traffic which is above 40Kbps, give significant impact to latency performance. As observe in Fig. 4, average latency increases significantly as traffic load increase. Heavy traffic caused much packet to be generated per unit time. Since each user had limited available slots, there are several packets need to be queued before transmission. This situation had increased the queuing delay which then results in increasing packet latency. Due to this reason, shortest hop count is preferable since less hop count would provide less delay. However, above result had proven that selection based on hop count is not contributes in achieving better ETE delay performance. The proposed scheme produce better ETE delay by about $4.3 \%$ less than the shortest scheme since it considers link quality during selecting path. Good link quality provides more capacity, thus more bits can be transferred per unit time. Consequently, it would helps in reducing queuing delay at users. Hence, improve latency performance.

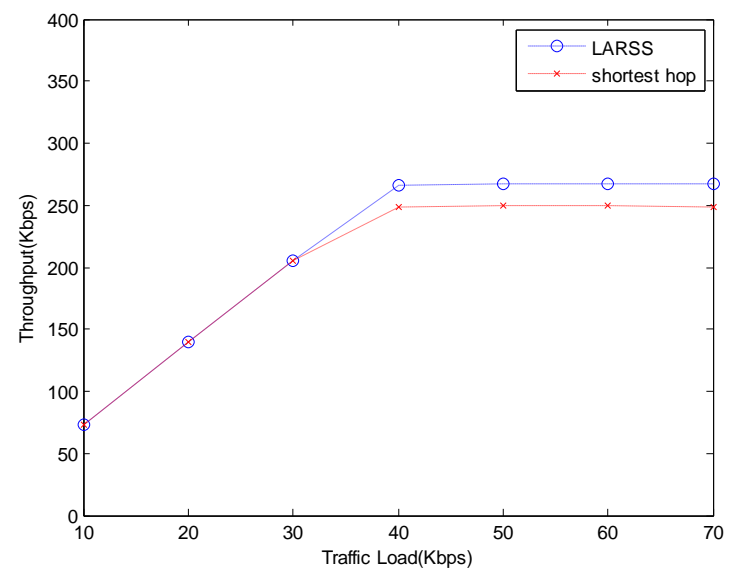

Fig. 3. Throughput performance comparison

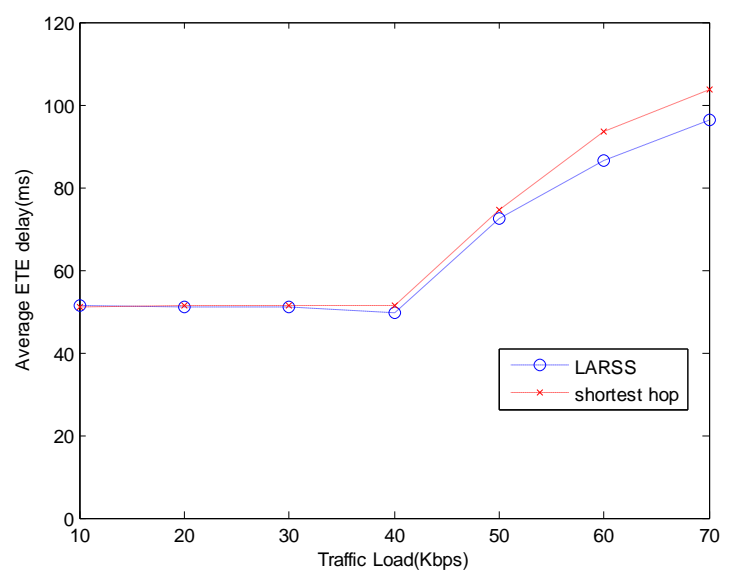

Fig. 4. Average end to end delay comparison

\section{CONCLUSION}

In this paper, we proposed route selection scheme suitable for non-transparent mode WiMAX MMR network. As working on multi-hop relay network which might lead to performance degradation as number of hops increase, we solve this issue by means of proposing LARSS which considered hop count and link quality as the route metric. The performance of proposed LARSS was evaluated through simulation. Simulation results had shown that the proposed LARSS outperformed the shortest path scheme by about $6.7 \%$ and $4.3 \%$ for throughput and end-toend delay performance, respectively. Our proposed LARSS provide much better throughput and less ETE delay during heavy traffic load. In large network, there is a possibility that congestion might happen. Thus, the availability of resources at each RS should be considered as well during making a route selection. This issue will be left for future work. 


\section{ACKNOWLEDGMENTS}

The authors wish to thank the Ministry of Higher Education (MOHE), Universiti Teknologi Malaysia (UTM), MIMOS Berhad and Research Management Centre (RMC), UTM for the Sponsorship and Telematic Research Group (TRG), Universiti Teknologi Malaysia for the support and advice. This work is supported by MOHE under grants Q.J130000.7123.01F.01H35.

\section{REFERENCES}

[1] IEEE Standard for Local and Metropolitan Area Networks Part 16: Air Interface for Fixed and Mobile Broadband Wireless Access Systems Amendment 1- Multi-hop Relay Specification. 2009. IEEE Std P802.16j/D9

[2] Wang, K., Peng, M., Wang, W. 2007. Channel Aware Adaptive Resource Allocation in Two-hop Wireless Relay Networks. In IEEE International Symposium on Personal, Indoor and Mobile Radio Communication

[3] Lee, K., Lee, H. 2009. A Joint Bandwidth Allocation and Routing Scheme for the IEEE 802.16j Multi-hop Relay Networks. In IEEE International Conference on Information Networking

[4] Dong, J.S., Ju, W.J. 2010. A Path Selection Scheme Considering Traffic Load for IEEE 802.16j Mobile Multihop Relay Networks. In International Conference in Wireless Communications, Networking and Mobile Computing

[5] Shih, K.P., Wang, S.S., Lien, C.Y. 2009. A High Spectral Efficiency and Load-Aware Metric for Path Selection in IEEE 802.16j Multi-hop Relay Networks. In IEEE Symposium on Computers and Communication, 61-66.

[6] Deepesh, M.S., Sung, H.L., Sung, C.K, Young, B.K. 2007. New Approaches for Relay Selection in IEEE 802.16
Mobile Multi-hop Relay Networks. In M. Kermarrec, L. Bouge and T. Priol(eds.) Euro-Par 2007. LNCS, vol. 4641, 950-959. Springer, Heidelberg

[7] Nurulashikin Satiman, Rozeha A. Rashid, Norsheila Fisal and et.al. 2011. A SNR-based Route Selection Algorithm for WiMAX Mobile Multi-hop Relay Networks. In World Wireless Research Forum 2011. Doha, Qatar

[8] Soldani, D. Multi-hop Relay Networks. In WiMAX New Developments, Upena D Dalal and Y P Kosta (Ed.)

[9] Genc, V., Murphy, S.,Yu, Y., Murphy, J. 2008. IEEE802.16j Relay-based Wireless Access Networks: An Overview. In IEEE Wireless Communication, 56-63

[10] Seo, S.O., Kim, S.J., Kim, S.Y., Kim, Y.I, Lee, H.W., Ryu, S. Cho, C.H. 2010. Relay Performance Analysis of TTR and STR Relay Modes in IEEE 802.16j MMR System. In ETRI Journal, Volume 32, Number 2, 230-240

[11]Theodore S. Rappaport. 2002. Wireless Communications Principle and Practice Second Edition. In Prentice Hall publisher. 120-125

[12] IEEE Standard for Local and Metropolitan Area Networks, Part 16: Air Interface for Fixed and Mobile Broadband Wireless Access Systems Amendment 2: Physical and Medium Access Control Layers for Combined Fixed and Mobile Operation in Licensed Bands and Corrigendum. 2005. IEEE Std 802.16e-2005

[13] Shu'aibu, D.S., Yusof, S.K.S. 2011. Link Aware Earliest Deadline Scheduling Algorithm for WiMAX. In :International Journal of Communication Networks and Information Security, Volume 3, Number 1, 83-88 